www.jmscr.igmpublication.org

Index Copernicus Value: 79.54

ISSN (e)-2347-176x ISSN (p) 2455-0450

crossref DOI: https://dx.doi.org/10.18535/jmscr/v7i6.103

\title{
An Unusual Complication of Fibroid during Postpartum Period: Degenerated Fibroid Mimicking Postpartum Uterine Haematoma
}

\author{
Authors \\ Manika Agarwal*, Lungan Rongmei, Subrat Panda \\ Department of Obstetrics and Gynaecology, NEIGRIHMS Hospital, Shillong, Meghalaya, India \\ *Corresponding Author \\ Manika Agarwal \\ Department of Obstetrics and Gynaecology, NEIGRIHMS Hospital, Shillong, Meghalaya, India
}

\begin{abstract}
A case of 38 years female presenting in emergency department with lower abdominal pain, fever and vomiting on day 13 puerperium. She developed this symptoms 4-5 day post caesarean. On examination patient was febrile with temperature 101.8 degree farenheit and tachypneic . She was also having tachycardia and was pale. On abdominal and vaginal examination uterus was 14 weeks size, mildly tender. After admission patient was started with supportive treatments and antibiotics. On MRI examination, it showed a posterior uterine wall haematoma with mass effect. As patient fever was not responding to higher antibiotics and as posterior uterine wall collection may be the cause of fever, so patient was prepared for emergency laparotomy on $4^{\text {th }}$ October. Intraoperatively there was a solid-cystic mass in posterior uterine wall, which was eneucleated and send for HPE.
\end{abstract}

\section{Introduction}

Uterine fibroid, one of the most common benign tumours of reproductive age. Pregnancy is characterized by increase in size of fibroid, commonly in first trimester. Degenerative changes in a fibroid commonly occur during pregnancy when the size of the tumor outgrows its blood supply leading to necrosis of the tissue in it.It is known as red or carneous degeneration of fibroid characterised by pelvic pain, fever and elevation of white blood cell count ${ }^{[1]}$. But, most uterine fibroids either stop growing or decrease in size postpartum $^{[2]}$. Here we present a case of 38 years female who was referred to our institute with lower abdominal pain, fever and posterior uterine wall cyst on day 13 post LSCS.

\section{Case Report}

A case of 38 years female P1L1 at day 13 post LSCS came to emergency department with complains of lower abdominal pain, fever and vomiting. She developed these symptoms 4-5 days after delivery. However, there was no history of bleeding per vagina. She had emergency LSCS for obstructed labour 13 days back. Patient was stable at the time of discharge. On examination, patient was febrile with temperature 101.8 degree Farenheit and tachypneic. She had heart rate of $110 / \mathrm{min}$, respiratory rate of $24 / \mathrm{min}$ and was pale. On abdominal examination her abdomen was soft with normal bowel sounds, longitudinal LSCS wound was healthy, a firm mass $(8 \times 7) \mathrm{cm}$ was palpable which was arising from pelvis, mildly 
tender. On vaginal examination uterus was 14 weeks size, mildly tender, bilateral fornices were free and non-tender. Rest of her physical examinations was normal. All fever investigations were negative. Her urine and blood culture were also negative. All routine investigations were in normal range except for hb $5.6 \mathrm{gm} / \mathrm{dl}$ and increased white blood cell counts $\left(15.8 \times 10^{3}\right)$. After admission in ward, patient was started with supportive treatments and injectable antibiotics. Two units of packed cells was transfused. Repeat hemogram showed hb has increased to $8.6 \mathrm{gm} / \mathrm{dl}$ but white blood cells count was in increasing trend. Ultrasonography showed uterine posterior myometrialcystic lesion with internal debris suggestive of degenerative uterine fibroid with secondary infection or posterior uterine wall haematoma. So for confirmation MRI pelvis done, it showed uterine intramural posterior wall haematoma $(11 \times 8 \times 9) \mathrm{cms}$ with mass effect. As patient fever was not responding to higher antibiotics and as posterior uterine collection may be the cause of fever, so patient was prepared for emergency laparotomy on $4^{\text {th }}$ October. Consent was taken and patient was explained the risk of PPH and hysterectomy. Intraoperatively uterus was 14 weeks size and (8x10) cm mass was noted on posterior wall of uterus- solid cystic consistency. Enucleation of the mass done and send for HPE. Cystic fluid aspirated from mass was also sent for culture and sensitivity.

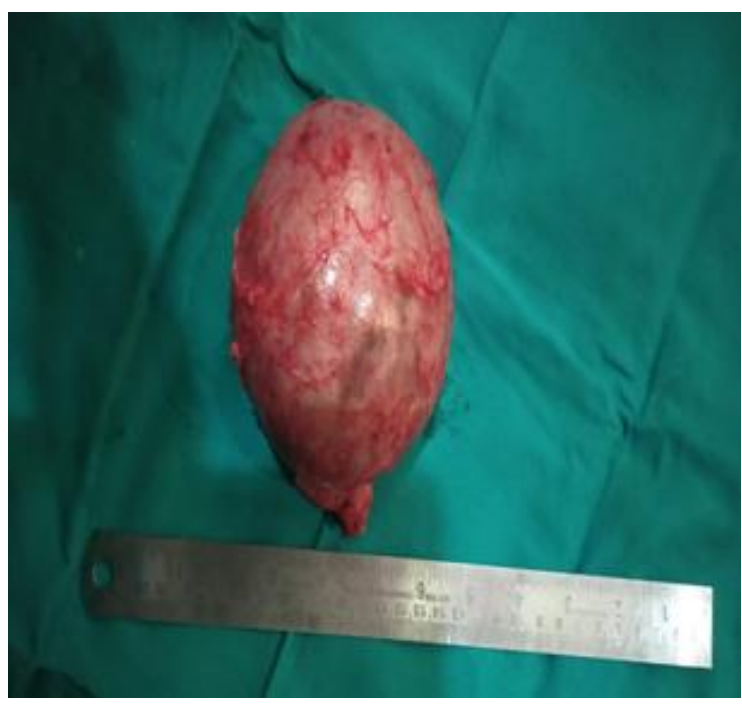

Fig 1. Picture of eneucleated mass.

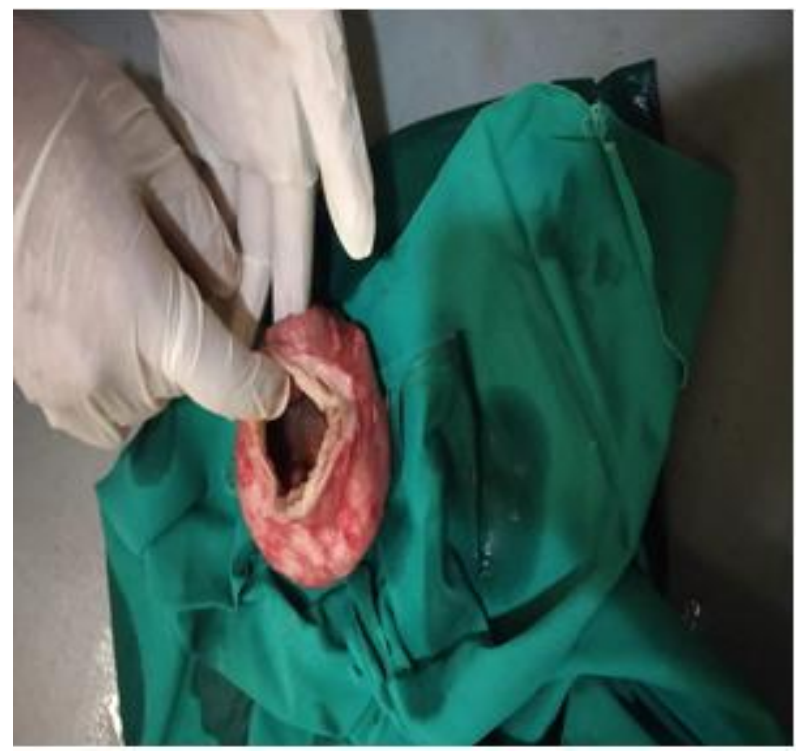

Fig 2.Cut section of eneucleated mass.

Post-operatively patient was improving, looking better. Fever subsided from day 3 postoperatively. But on day 4 of operation, patient complains of pain and swelling of legs.USG Doppler of lower limbs shows bilateral leg cellulits. It was treated with $\mathrm{MgSO} 4$ dressing and antibiotics. Slowly patient was improving with no more fever and bilateral limbs swelling has also decreased after treatment and patient was discharged in a healthy condition.

HPE of specimen shows features of leiomyoma with central part undergoing degenerative and cystic change with hyalinization and calcification

\section{Discussion}

Uterine fibroids is one of the most common benign tumours of the genital organs in women of reproductive age. Myomas are observed in about $3-12 \%$ of pregnant women ${ }^{[4]}$. The majority of fibroids do not change their size during pregnancy, but one-third may grow in the first trimester ${ }^{[5]}$. Fibroids during pregnancy can cause many perinatal complications, such as bleeding in pregnancy, miscarriage, pain due to red degeneration, malpresentation, preterm labor, premature rupture of membranes, placental abruption and obstruction of delivery and are associated with higher incidence of cesarean section, operative vaginal delivery, uterine atony and postpartum hemorrhage ${ }^{[3]}$. 
Red degeneration is a hemorrhagic infarction of the uterine leiomyoma, which is a well known complication, especially during pregnancy. Red degeneration occurs in $8 \%$ of tumors complicating pregnancy, although the prevalence is about $3 \%$ of all uterine leiomyoma ${ }^{[6]}$. It present with symptoms such as abdominal pain, tenderness, fever and increased white blood cell count. But most fibroids stop growing or decline during the puerperium $^{[3]}$. Symptomatic fibroid degeneration during puerperium, therefore, is rare, as happened in our case.This is the first reported case in literature of degenerated fibroid immediately after an emergency caesarean section. On search of literature we found one case report where patient present 3 months after vaginal delivery with same symptoms of pain, fever and leucocytosis. Ultrasound and MRI findings were inconclusive. After failure of antibiotic treatment patient was taken for exploratory laparotomy which showed broad ligament fibroid with degeneration ${ }^{[1]}$

\section{Conclusions}

This was an unusual case of fibroid with degeneration presenting with puerperal pyrexia when conservative management failed to treat the patient, a well timed surgical approach helped to save the patient's life.

Conflict of Interest: There is no conflict of interest.

Informed Consent: We have taken informed consent of patient for taking photograph and publishing the case in journal.

\section{References}

1. Neha V. Bhave, P. K. Shah, Hemangi Chaudhari. An unusual presentation of degenerating fibroid. Int $\mathrm{J}$ Reprod Contracept Obstet Gynecol. 2016;5(2): $582-584$
2. Natalia Pricea, Kailash Nakadea, Sean T. Kehoeb. A rapidly growing uterine fibroid postpartum. $\mathrm{Br} \mathrm{J}$ Obstet Gynaecol. 2004;111:503-505.

3. Sparić R.Uterine myomas in pregnancy, childbirth and puerperium. Srp Arh Celok Lek. 2014;142(1-2):118-24.

4. Parazzini F, Tozzi L, Bianchi S. Pregnancy outcome and uterine fibroids. Best Pract Res Clin Obstet Gynaecol. 2016;34:74-84.

5. Hee Joong Lee, Errol R Norwitz, and Julia Shaw. Contemporary Management of Fibroids in Pregnancy. Rev Obstet Gynecol. 2010; 3(1): 20-27.

6. Seung Chul Han, Man-Deuk Kim, DaeChul Jung, Myungsu Lee, Mu Sook Lee, Sung Il Park, Jong Yun Won, Do Yun Lee, and Kwang Hun Lee. Degeneration of Leiomyoma in Patients Referred for Uterine Fibroid Embolization: Incidence, Imaging Features and Clinical Characteristics. Yonsei Med J. 2013;54(1):215-219. 